Pure \& App I. Chem., Vo1 56, No. 12, pp 1677-1696, 1984. Printed in Great Britain.
$0033-4545 / 84 \$ 3.00+0.00$ Pergamon Press Ltd.

(C) 1984 IUPAC

\title{
INTERFACIAL ADSORPTION AND REACTIVITY OF MATERIALS
}

\author{
E D Hondros
}

Division of Materials Applications, National Physical Laboratory, Queens Road, Teddington, TW11 OLW

\begin{abstract}
In the context of the theory of materials design, we consider the role of adsorption - at free surfaces and intercrystalline interfaces - on kinetic phenomena which in turn influence significantly the mechanical and processing properties of materials. In particular, we discuss gas phase surface hardening reactions, grain boundary mass transport and precipitate ripening processes, with a view to establishing guidelines for improved performance, through an understanding of the nature and effects of adsorbing species.
\end{abstract}

\section{INTRODUCTION}

Here we are concerned with materials systems in relation to their high temperature applications or processing, including inorganic as well as metallic materials. In considering chemical reactivity in polycrystals, the aspect which sets them apart from classical homogeneous reaction rate theory is that heterogeneity dominates these reaction processes: this arises from the presence of defect structures - point defects, two-dimensional defect structures such as interfaces and surfaces, and three-dimensional defect structures such as particles and second phases distributed within the matrix of the solid. We are struck again by further evidence of heterogeneity in chemical reactivity through the numerous phenomena involving nucleation and growth of product phases, such as precipitates, or even of more exotic metallurgical forms such as gas bubbles or micro-cavities strung along grain boundaries, all of which influence mechanical properties and processing efficiency.

Since chemical reactivity between a solid and a fluid phase invariably involves the interface between these phases, the two-dimensional diffusion along the interfaces or the diffusive transport of matter across them are the important kinetic events which control the rate of interfacial chemical reactions. Thus the basic properties of interfaces their structure, microchemistry and energetics are very important in the description of the chemical reactivity in which these interfaces participate.

We use the term 'interface' here quite liberally to indicate the two-dimensional surface of separation between regions of different chemistry or state of orientation in engineering materials. The complexity of interface types in a normal alloy is indicated schematically in Fig. 1. Thus the solid/vapour interface or free surface can manifest itself either as the external material surface or the surface of the oxide layer on a metal, or the surfaces of voids or gas bubbles trapped within. The inter-crystalline boundaries are the interfaces between chemically identical regions that have different crystalline orientation, whereas the interphase boundaries separate regions of different constitution as well as crystallinity. From a macroscopic point of view, the thermodynamic and kinetic properties of these interfaces can be described by a similar formalism in spite of widely differing, often very complex structures at the atomic level. The fundamental kinetics of atoms at any of these features can influence profoundly the rates of mass transport, which in turn lead to important phenomena such as precipitate stability, sintering and oxidation.

A central phenomenon which pervades all of surface chemistry is that of the adsorption of chemical species at interfaces, which may occur by the formation of strong chemical bonds or by a weaker physical attachment of the adsorbate molecules. In general, this leads to adsorbed films of only atomic dimensions. The parallelism in behaviour of the various interfaces in connection with adsorptive processes is indicated in Fig. 2 . This shows on the same plot the variation in interfacial free energy of iron as a function of tin content in the bulk, for a liquid surface, a solid surface and a grain boundary ${ }^{(1)}$. 


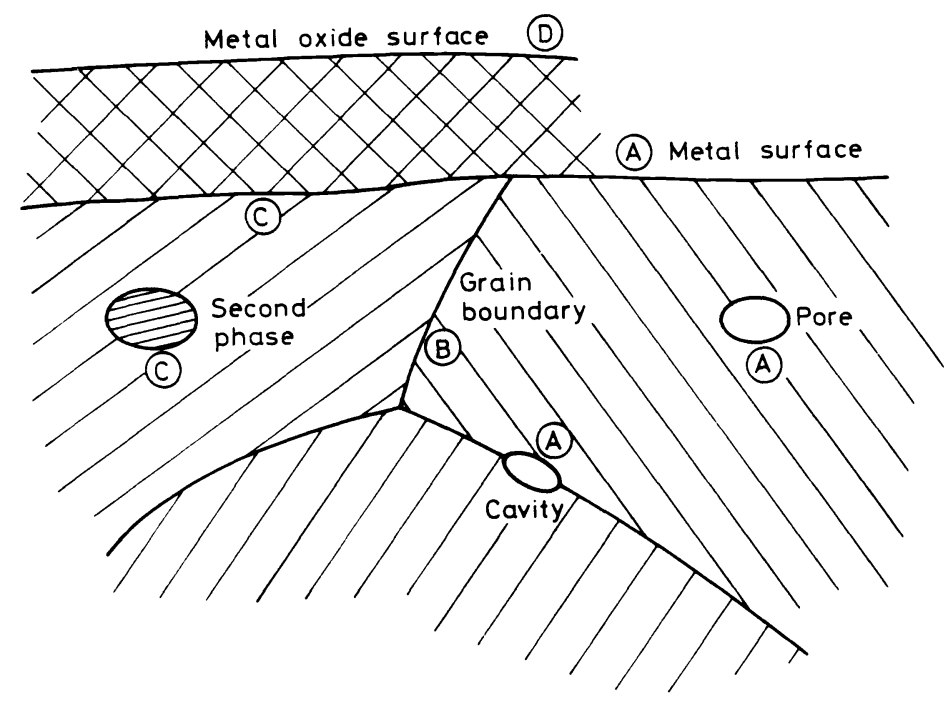

Fig. 1 Schematic representation of polycrystalline interfaces which may influence chemical reactivity

The isotherms show a similar trend, namely an early rapid fall followed by a gradual weakening effect. The magnitude of the initial fall in free energy depends on the interface and the isotherms gradually approach a constant but different level. This is typical of behaviour encountered in many such binary systems.

This provides a valuable insight into interfacial adsorptive behaviour because the change in energy is related to the amount enriched at the interface through the classical Gibbs adsorption theorem, which describes in its general form adsorption in systems of the highest chemical complexity. For the simple binary systems displayed in Fig. 2, the Gibbs equation appropriate to dilute conditions has the simple form,

$$
\left.\frac{d \gamma}{d \ln x_{c}}\right|_{T}=-\operatorname{RT} \Gamma_{S}
$$

where $\Gamma$ is the equilibrium excess solute at the interface (expressed as $\mathrm{mol} / \mathrm{m}^{2}$ ), $\gamma$ the interfacial free energy and $x_{c}$ the molar concentration of the second species.

From Equation 1 the amount of interfacial excess is derived and transformed into equivalent monatomic layers. In this specific case, the behaviour of the liquid surface is ideal in that it shows the approach towards saturation adsorption of tin of about a single monatomic layer at the iron surface. This is typical of a large number of binary systems studied, even for the solid state. In the case of tin in solid iron, the interfacial excesses do not show saturation monolayer adsorption, in fact they form multiple layer segregation. However, for most solid state systems that have been studied so far, such as oxygen and phosphorus in iron, saturation occurs at levels somewhat below a monatoric layer. Not only can the relation between adsorption and change in interfacial free energy be demonstrated for all interfaces in solids, but also the various detailed laws of surface adsorption - sub-monolayer, multi-layer adsorption, interactive adsorption, can occur in analogous formalisms at all interfaces of interest in polycrystalline materials. 


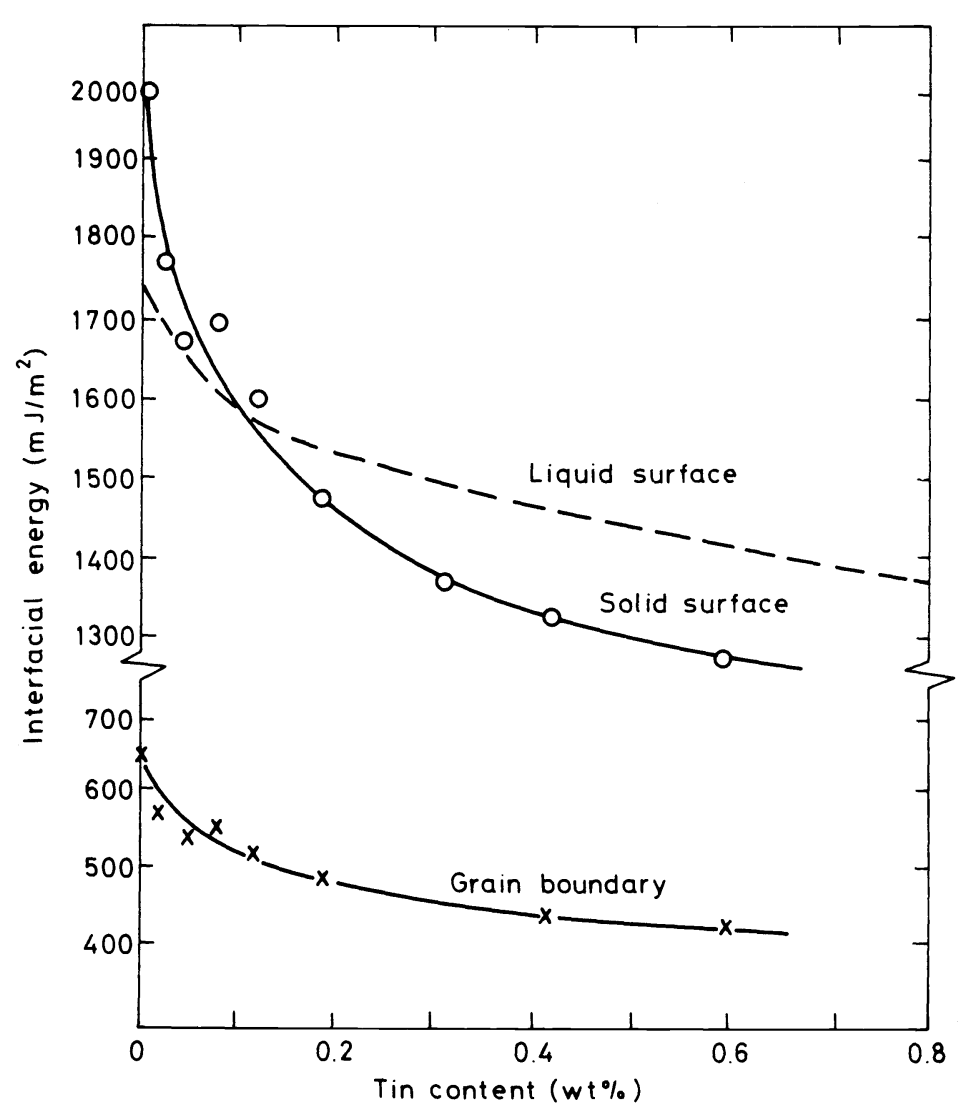

Fig. 2 Variation of free energies of interfaces in solid and liquid iron with increasing tin content in the bulk

In this paper we examine then, how does this ubiquitous adsorption at interfaces in engineering materials influence the reactivity of the solid? We should emphasise that not only is this important in understanding reaction rate processes, but it is also of considerable technological interest, both in materials processing and in materials performance in service conditions. We shall demonstrate, using examples taken from work in the literature, that the effects of interfacial adsorption on reactivity are often significant and dominating. Our enquiry will take us further - how can such understanding be used as a control or a modifier of chemical reactivity?

As part of a plan for materials design, such potential control of behaviour in reactive environments can be used to modify the mechanical or physical properties. For example, in materials used in high temperature plant, as in turbine applications, an incremental improvement in service performance can lead cumulatively to large economic benefits. The materials scientist faces the task of a sustained improvement in performance of the materials used by industry through a control of bulk microstructural and metallurgical variables. Thus, through a deeper understanding of the interactions between the adsorption process, chemical reactivities and the mechanical properties, we may provide a potential tool with which to fine-tune engineering performance. Hence the technologist's aim in the context of this paper is improvements and control of processability, as in powder metallurgy and sintering, as well as improvements in performance through, for example, surface protection and the avoidance of problems such as creep cracking.

\section{ADSORPTION BEHAVIOUR AT MATERIALS INTERFACES}

Considering as an archetypal case the effect of phosphorus in dilute bulk content on the 
surface and grain boundary energies of iron (2), the basic behaviour is shown in Fig. 3. This typifies the behaviour of a number of species such as oxygen. From the slopes of the surface tension isotherms, the calculated surface and grain boundary excesses are superimposed on the same Figure. This provides information on the interfacial excess in absolute terms. Thus, at the saturation part of the isotherm, the coverage of phosphorus on the free surface is $1.4 \times 10^{9}$ atoms $/ \mathrm{m}^{2}$ of surface, which corresponds to about 0.75 atoms of phosphorus per outermost atom of iron. In the case of the grain boundary, the excess is about one third of a monatomic layer, assuming the phosphorus atoms to be hard spheres spread out evenly and compactly across the interface.

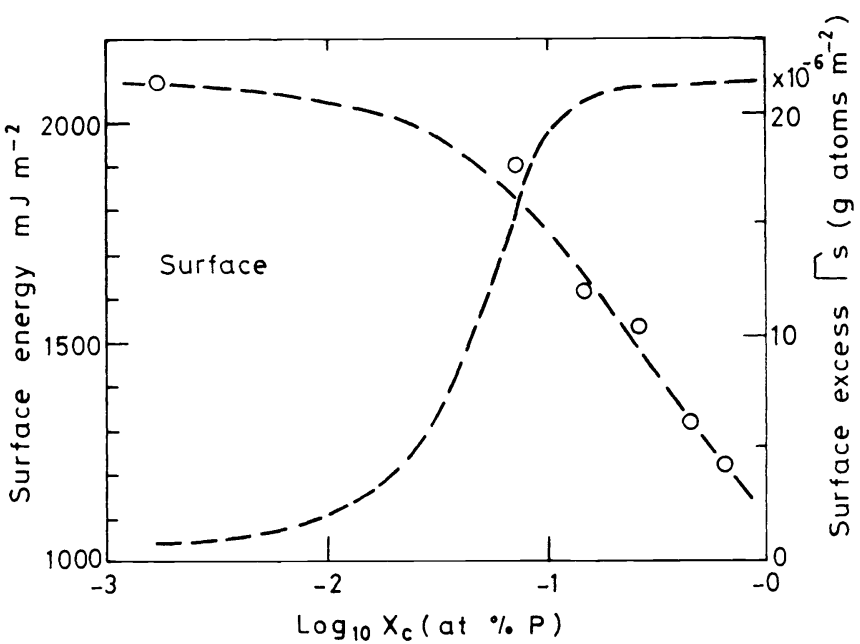

(a)

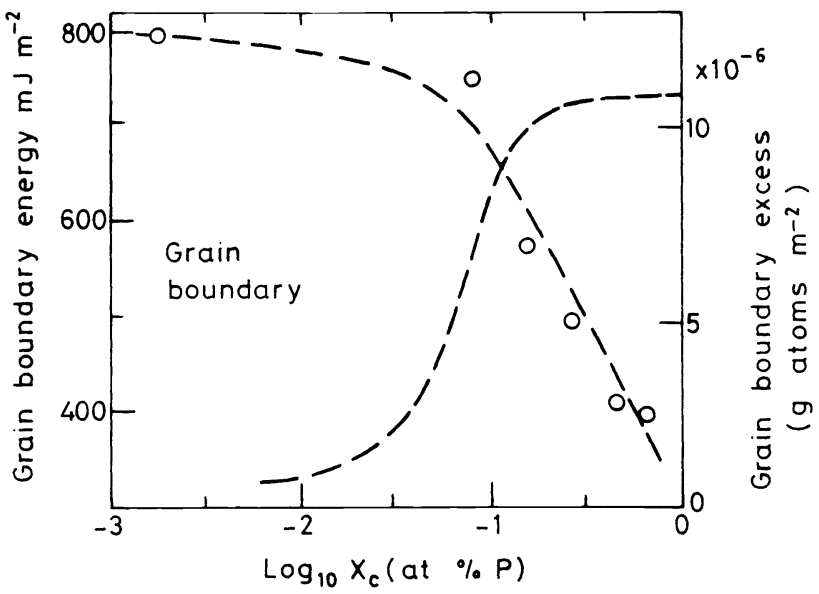

(b)

Fig. 3 Variation in interfacial free energy and adsorption in iron with increasing bulk content of phosphorus: Top figure: Surfaces Lower figure: Grain boundaries

The experimental methods leading to these measurements are laborious and difficult. However, they supply the only reliable information appropriate to polycrystalline materials at very high temperatures, approaching the melting point. Information of this nature is probably the most relevant to a consideration of reaction processes involving the transfer of atoms across interfaces. Until the advent of the many sophisticated surface spectroscopies for measuring surface composition, this Gibbs adsorption approach represented the only quantitative means available for detecting and measuring adsorption. In the meantime a wide range of analytical techniques, including Auger Electron Spectroscopy and Secondary Ion Mass Spectroscopy have been developed and provide direct 
information on the surface composition of solids. The techniques have high detectability and sensitivity, even in multi-component adsorption and procedures for quantifying the chemical analyses are being developed. However, because they must operate in conditions of very high vacua, their useful range of application lies more at the lower temperatures than those considered here. It is not within the scope of this paper to discuss the various techniques; for the interested reader we refer to a recent detailed review by Hondros and Seah ${ }^{1}$, which also treats the basic phenomena of interfacial adsorption in polycrystalline materials.

The concepts introduced by surface chemists to describe adsorption at solid surfaces can be formally extended to account for adsorptive processes at all interfaces. In this way, many familiar adsorption isotherms - the Langmuir, the Fowler and other well-known forms can be applied to describe the equilibrium concentration of species at internal interfaces as a form of adsorptive behaviour. Thus, the grain boundary segregation analogue of Langmuir or saturation monolayer adsorption is: 3

$$
\frac{X_{b}}{X_{b}^{O}-X_{b}}=\frac{X_{c}}{1-X_{c}} \exp \frac{-\Delta G}{R T}
$$

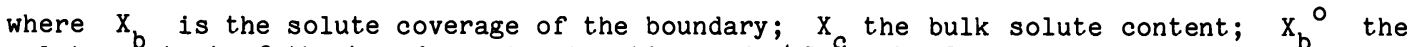
solute content of the boundary at saturation and $\Delta G$ is the free energy of segregation per mole of segregant. This derivation assumes no interaction between the adsorbing species and in this form, the McLean expression above, obtained independently by a statistical mechanics approach, is identical to the classical Langmuir equation. Here, $X^{\circ}$ is approximately one third of a monatomic layer, corresponding to the grain boundary excess free volume. The equation expresses the basic features of this common type of boundary adsorption: the segregant level increases as a function of bulk solute concentration and decreasing temperature. Segregation measurements using both the surface spectroscopies and the Gibbs interfacial energy approaches have been reported and these, on analysis, obey the form of Equation 2 for both free surfaces and grain boundaries.

In a similar way, different isotherms can be derived in analogous form in order to describe more complex adsorptive behaviour. In particular, we have demonstrated the validity of the BET theory in predicting multi-layer segregation at grain boundaries, as manifested in the system iron/tin. Even more complex interfacial segregation types of behaviour may be analysed by this analogue process; for example, cases where there exists a spectrum of binding energies of adsorption sites, or where there exist interactions between the segregating species (Fowler adsorption). In the case of the segregation of selenium to the grain boundaries in iron, the segregation is so intense that when analysed in terms of the Fowler model, there are strong indications that a two-dimensional phase can be formed at that interface.

The propensity for species to adsorb at an interface varies over many orders of magnitude. The enrichment ratio $(B)$ expresses the intrinsic segregation propensity in a given system. This is defined as the ratio between the interfacial concentration $X_{s}$, (in mole fraction of a monatomic layer) and the bulk concentration $X_{\text {(in }}$ mole ${ }^{s}$ fraction), $\beta=\frac{X_{S}}{X_{c}}$. This is related to the change in interfacial free energy by

$$
\beta=\frac{X_{S}}{X_{c}}=\frac{\Gamma_{S}}{\Gamma_{S}^{0} X_{c}}=-\left.\frac{1}{R T \Gamma_{S}^{0}} \frac{d \gamma}{d X_{C}}\right|_{T}
$$

where $\Gamma_{\mathrm{S}}^{0}$ is the calculated quantity of solute in mol/m contained in one monatomic layer.

By expressing all enrichment data in this form, patterns of enrichment behaviour for a wide range of systems have been compared and inter-related.

There exist already many evaluations of $\beta$, both in binary and in more complex systems. For grain boundaries, these have been compiled into a single logarithmic relation by plotting $\beta$ as a function of the inverse of the solid solubility of the segregating species at the temperature of measurement, $x_{c}$. The least squares fit to the data yield the relation: 


$$
\beta=\frac{5}{\mathrm{X}_{\mathrm{C}_{0}}}
$$

Thus, from a knowledge of the solid solubility, available from the constitutional phase diagram, $\beta$ may be estimated directly. This is not only a particularly useful guideline for segregation behaviour, but it can be used for evaluating $\beta$ in systems which are difficult to study by direct analysis. Thus, in steels, the low solubility elements such as $\mathrm{S}, \mathrm{Se}, \mathrm{Te}$ and $O$ have the highest enrichment factors.

\title{
3 INTERFACIAL REACTIVITY AND TECHNOLOGICAL PHENOMENA
}

Adsorption at interfaces influences the bulk mechanical and processing properties of materials in an indirect manner, through the effect on some physico-chemical property. We distinguish certain broad behavioural types. These are: kinetic, cohesive, energetic and electrochemical. There are many important cases which fall in the latter three categories through which interfacial microchemistry can have a remarkable effect on metallurgical properties: for example, in reduced grain boundary cohesion at low temperatures which is reflected in metallurgical problems such as temper embrittlement. Here we will be concerned only with the phenomena which fall into the first category.

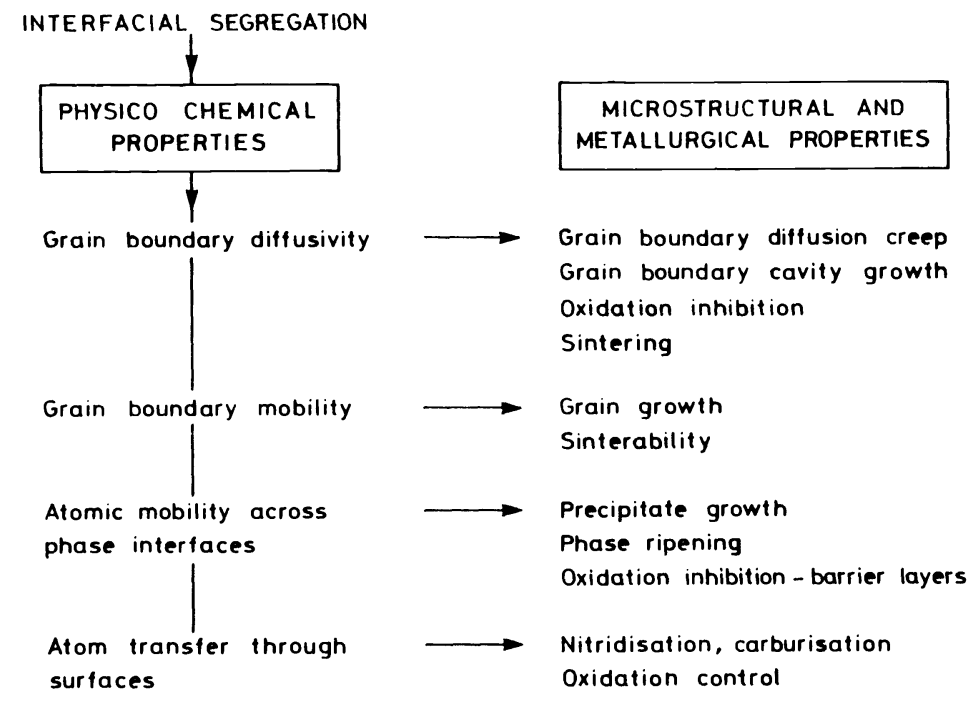

Fig. 4 Schematic diagram indicating interface reaction controlled materials phenomena

\begin{abstract}
Adsorption-affected interfacial reactivity must involve the fundamental kinetics for transport of atoms across and along interfaces. Hence it is not surprising that the cases that we refer to here involve phenomena at high temperatures. This does not gainsay the fact that at low temperatures, in particular in electrochemical processes, adsorption at interfaces may affect the rate of the reaction in solution: there is evidence, for example, that the presence of certain impurities such as sulphur which accumulate at the reacting interface of a metal in an aqueous corroding medium may affect the rate of that reaction. We do not consider such cases here because the basic impurity segregation event is a non-equilibrium phenomenon such as a statistical accumulation of the impurity species. We consider only those phenomena at high temperature in which the chemical reactivity is affected by an adsorptive process which can exist at equilibrium at that temperature, according to the laws of segregation indicated in Equations 2 and 3 .
\end{abstract}

The various kinetic phenomena at high temperature occurring during processing or during the performance of a material, are indicated schematically in Fig. 4. Interfacial adsorption will affect those physico-chemical properties indicated in this schema; each of these basic properties, for example grain boundary diffusivity or atomic mobility across interfaces, will have an impact on one or several microstructural and metallurgical properties of technological interest. We note that each of the metallurgical properties can be affected through changes in one or several of the basic physico-chemical properties: thus, the integrity of an oxide layer may be affected by the altered 
interfacial adhesion caused by the presence of adsorbed solutes; however, the same adsorbate acting as a barrier layer at the metal/oxide interface may affect the kinetics of ion transport across that interface and, in addition, the same solute by adsorbing at the grain boundary of the oxide may change the rate of transport of matter along that interface.

Many examples have been reported in the literature which fall into the broad synthesis of Fig. 4. In some cases, the effect of the adsorption was a chance observation which was noted to produce erratic results. We do not attempt here an exhaustive review of all the cases that can be described in terms of adsorption-controlled reactivity, but indicate the more important studies which will be discussed with reference to the concepts outlined briefly above.

\section{SURFACE MODIFICATION REACTIONS}

In physical metallurgical practice, there exist important heat treatment processes such as nitridation or carburisation which are carried out in gas phase equilibria and which involve the transfer of atoms from the vapour phase to the interior of the solid. Here, nitrogen and carbon are diffused into the surface layers of steel through carrier gases such as $\mathrm{NH}_{3}$ or $\mathrm{C}_{2} \mathrm{H}_{4}$ in controlled gas phase mixtures containing $\mathrm{H}_{2}$ or $\mathrm{H}_{2} \mathrm{O}$. The presence of nitrogen of carbon in these outer surface layers imparts specific property improvements, in particular fatigue and wear resistance. This is a common industrial process used in the manufacturing of components such as gears and shafts.

In terms of chemical reactivity, the basic steps are the catalytic dissociation of the carrier molecule at the free surface, followed by the penetration of the interstitial element into the interior surface layers. The converse of these processes, de-nitridation or de-carburisation also occurs, depending on the heat treatment conditions and environment, and by the principle of microscopical reversibility, the steps are the diffusion of the species to the surface, followed by its catalytic association with the gas phase and evaporation from the surface. In industrial practice the need is to enhance the rate of gasification of the surface layers, leading to benefits through a faster throughput of components. Conversely, the denudation of the surface layers of nitrogen or carbon will lead to degradation in properties and must be controlled.

There now exist many observations and data both for the liquid and the solid state which affirm that the rates of the metal/gas interface reaction depend strongly on the presence of surface species which may be present in the carrier gas phase or in the bulk of the solid as an alloying element or impurity. The observations are broadly consistent with the notions of the catalytic poisoning of surfaces by impurities; in a closely related way, adsorption at the free surface retards the rate of ingress or of expulsion of elements such as carbon and nitrogen.

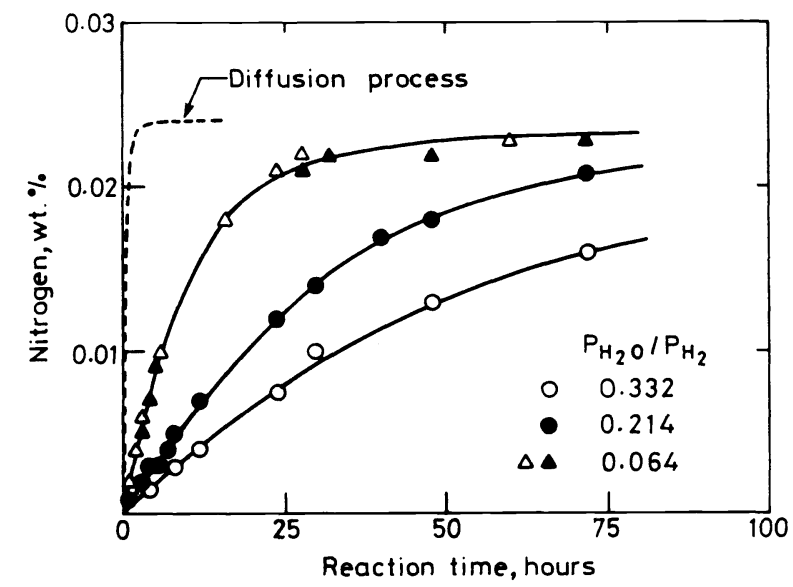

Fig. 5 Rates of nitridation of iron in gas phase mixtures containing low oxygen partial pressures (from Ref 5)

The earliest quantitative description of this process for the solid state is in the work of Turkdogan and Grieveson ${ }^{(5)}$, who showed that in the nitriding of steel in 
nitrogen/hydrogen mixtures at $1000{ }^{\circ} \mathrm{C}$, there exists a strong retarding effect of adsorbed oxygen. The data from these authors is reproduced in Fig. 5 where the rate of nitridation is presented for different values of the partial pressures of $\mathrm{H}_{2} \mathrm{O}$ and $\mathrm{H}_{2}$ which provide a free oxygen chemical potential. The broken curve in the diagram is that calculated for the simple diffusion controlled process for the entry of pure nitrogen in the steel and based on diffusive transport kinetics, a result which was later confirmed by experimental measurements under oxygen-free conditions. The effect is quite unequivocal - the higher the free oxygen potential in the gas stream, the greater is the reaction inhibited. The simplest explanation is based on the hypothesis of the poisoning of the surface by the surface active oxygen. In their model, the authors derived a first order reaction rate equation on the assumption that the oxygen completely covered the surface by an adsorbed layer. This model in turn predicted that the reaction rate should be inversely proportional to the oxygen partial pressure, which was confirmed experimentally.

The literature now contains copious data on the forward and reverse ${ }^{\mathrm{p}}$ rocesses for nitridation and carburisation, which were regiewed by Darken and Turkdogan $(6)^{\text {in }} 1970$ and later studies such as those by Fruehan $(7)$ who showed that decarburisation rates are markedly influenced by sulphur and phosphorus adsorption.

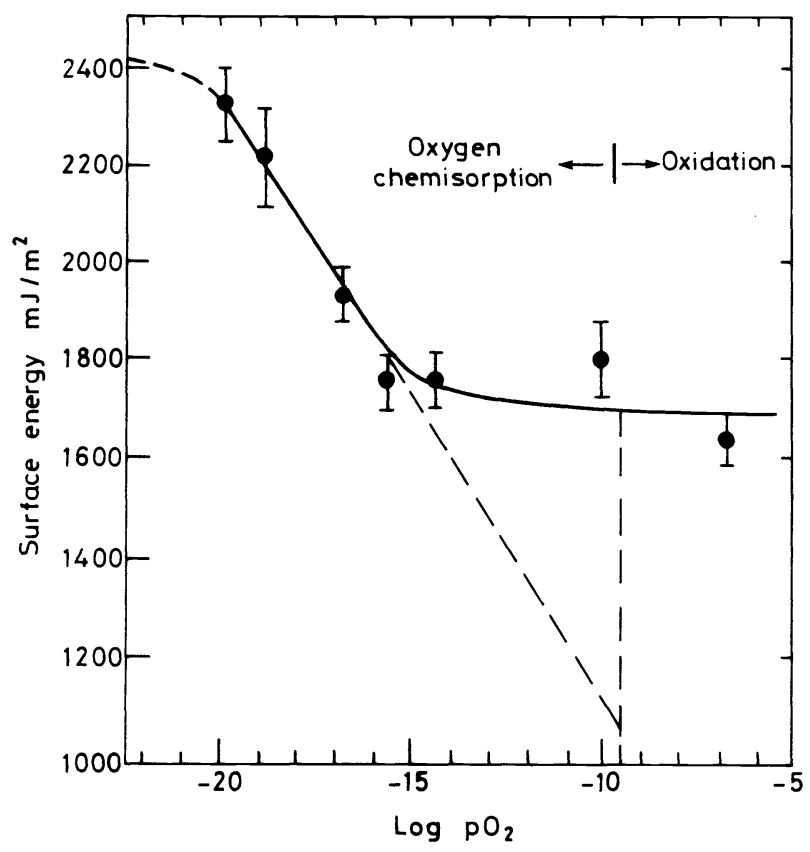

Fig. 6 The surface free energy of iron as a function of oxygen potential (from Ref 8 )

There is broad support of the adsorption model in the measurements by Hondros ${ }^{(8)}$ on the surface energy of iron as a function of oxygen potential of the environment, shown in Fig. 6. Here the downward sweep of the surface energy isotherm in the lower range of $\mathrm{pO}_{2}$ indicates an effective saturation of the surface with oxygen which at this temperature corresponds to about one third of a monatomic layer. Although the present data refer to the higher temperature phase, similar adsorption should hold at the lower temperatures of the work of Turkdogan and Grieveson. In general terms, extremely low values of oxygen potential in the gas stream can lead to strong oxygen adsorption at these temperatures. There are, however, some differences which are relevant to the detailed mechanisms for the reaction inhibited events. In Fig. 6, the oxygen saturation at sub-monatomic layer level persists over a very wide range of oxygen potential. The dashed part of the isotherm indicates the expected trend right up to the oxygen potential where bulk oxide formation is expected to set in. The data were explained in terms of a physical model consisting of a two-dimensional oxygen/iron mixed layer where the atoms are regrouped into a stable phase with the properties of an embryonic oxide. 


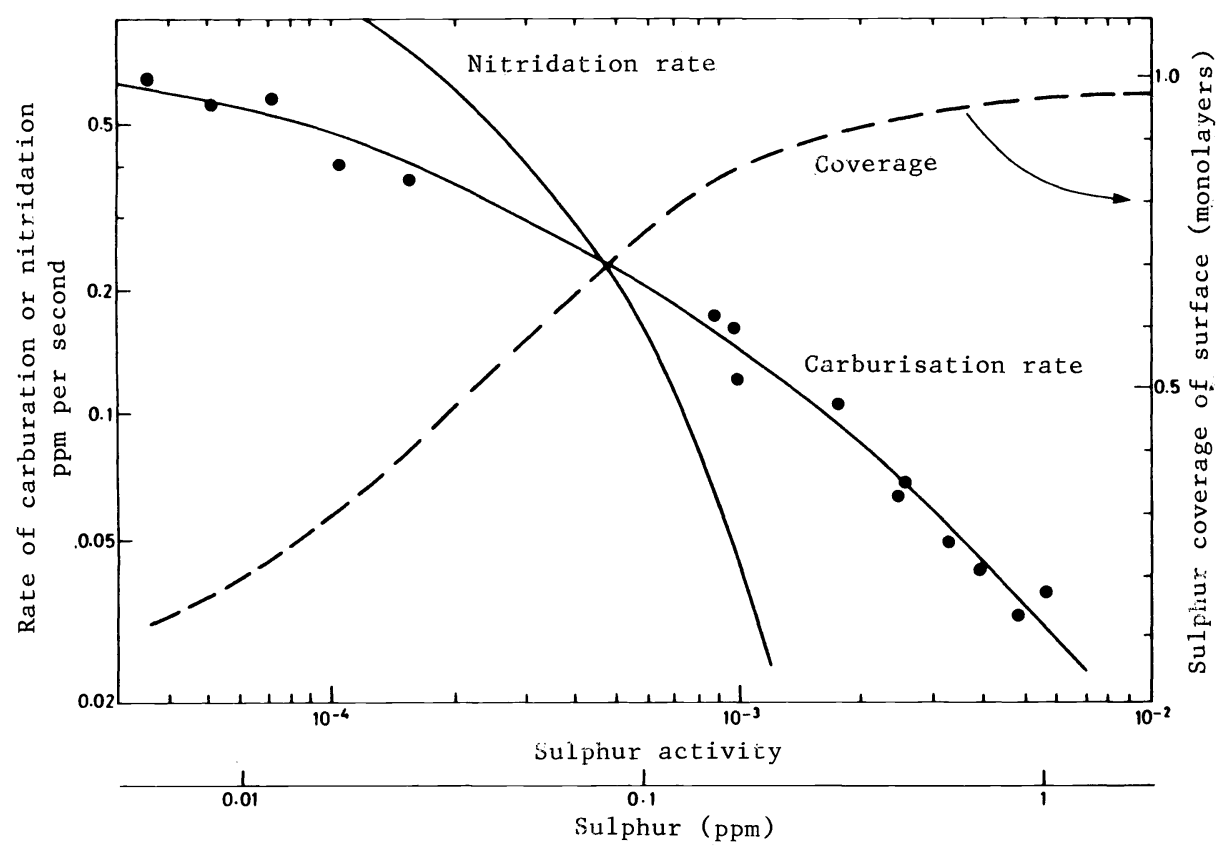

Fig. 7 Effect of sulphur at low concentrations on the rates of surface carburisation and nitridation (from Ref 9)

One of the difficulties in proposing a realistic model for the observed reaction behaviour is that there are usually no independent data for the adsorption of the poisoning species at the temperature of these experiments. We can predict from Equation 4 that sulphur should be another species with a high propensity to adsorb. Grabke et al ${ }^{2}$ studied the effect of sulphur impurities on the rate of nitridation and carburisation. Using gas flow mixtures of $\mathrm{H}_{2} \mathrm{~S} / \mathrm{H}_{2}$ to obtain the bulk sulphur content, the rates of nitridation and carburisation were measured; these are shown together on the same diagram in Fig. 7 . Thus at $850^{\circ} \mathrm{C}$, the nitrogen uptake decreases rapidly at very low sulphur contents and similarly but to a lesser extent, for the rate of carburisation. The sulphur coverage of the surface as a function of bulk sulphur content is also indicated in the figure; this was deduced from a model of interface inhibited reactivity by assuming that the rate of dissociation of the molecule depends on the area of clean surface exposed at any time. The coverages, varying up to a single monatomic layer, are the levels to be reasonably expected. The detailed mechanism of the inhibitive process requires that the adsorbate coverage increases with increasing sulphur activity.

Similar strong effects of phosphorus segregation at the free surface of iron on nitridation rates have been measured by Hayes and Grieveson ${ }^{19)}$; again, this is supported by the strong, phosphorus adsorption measured independently and shown in Fig. 3. Later, Miller et al 11$)^{2}$ carried out comprehensive studies with a range of surfactant species and measured particularly strong effects with impurity contents of antimony, tin and arsenic. Typical data are shown in Fig. 8 which demonstrates the effect of increasing bulk content of antimony up to $0.08 \%$ on the rate of nitridation of iron at $550{ }^{\circ}$. The relative effects of four different adsorbing elements on the rate of nitridation of alpha iron are indicated in Fig. 9. This shows the particularly strong effect of goncentrations of antimony on the process. We note that Togashi and Nishizawa (12) have measured independently the remarkable effect of antimony on the rate of decarburisation of austenite in wet hydrogen.

We have attempted to systematize the data in the literature; however, these are not sufficiently relatable in terms of corresponding measurements and adsorption levels of the poisoning elements. We note the following facts: in one category of Group VI elements, oxygen and sulphur are extremely surface active and, in addition, they affect very strongly the nitridation and carburisation reactions; in another category of Group $V$ elements such as $\mathrm{Sn}, \mathrm{Sb}, \mathrm{P}$ and As which are surface active (but not as high as oxygen or sulphur) there are again strong measurable effects on the rate of nitridation; in a third category of potential surfactants, Miller et al (fi) showed that $\mathrm{Ga}$, In, $\mathrm{Bi}$ and $\mathrm{Pb}$ have no inhibiting effect on the nitridation of alpha iron. It is possible that the latter elements react with impurity constituents in the gas phase and form volatile compounds. Hence their potential surface activity cannot be demonstrated unless the environment purity is highly controlled. 


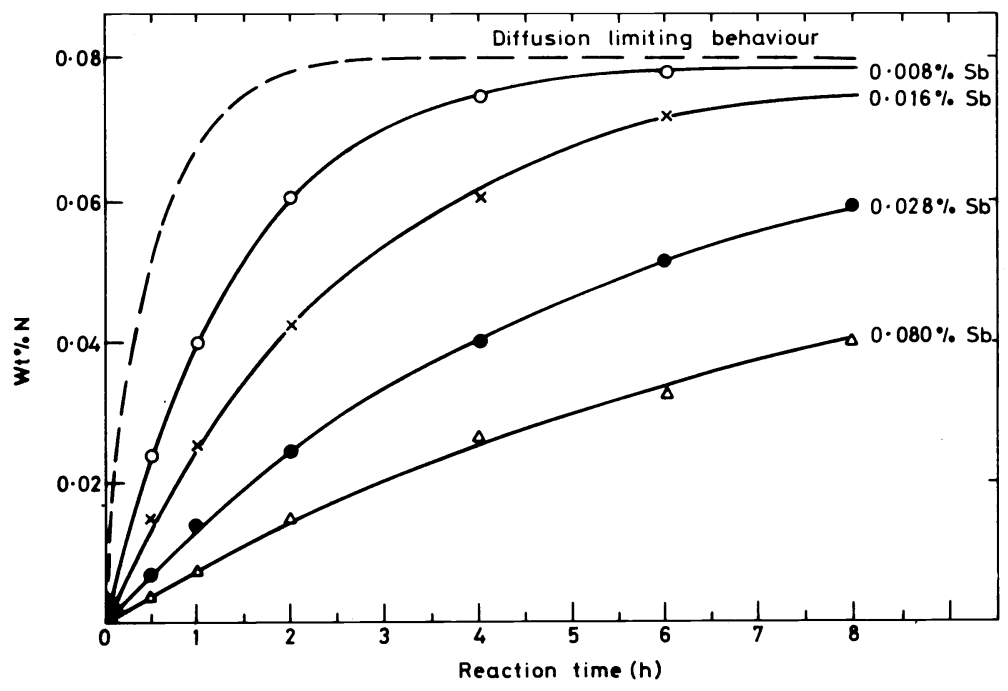

Fig. 8 Effect of antimony additions in bulk iron on the rates of surface nitridation (from Ref 11)

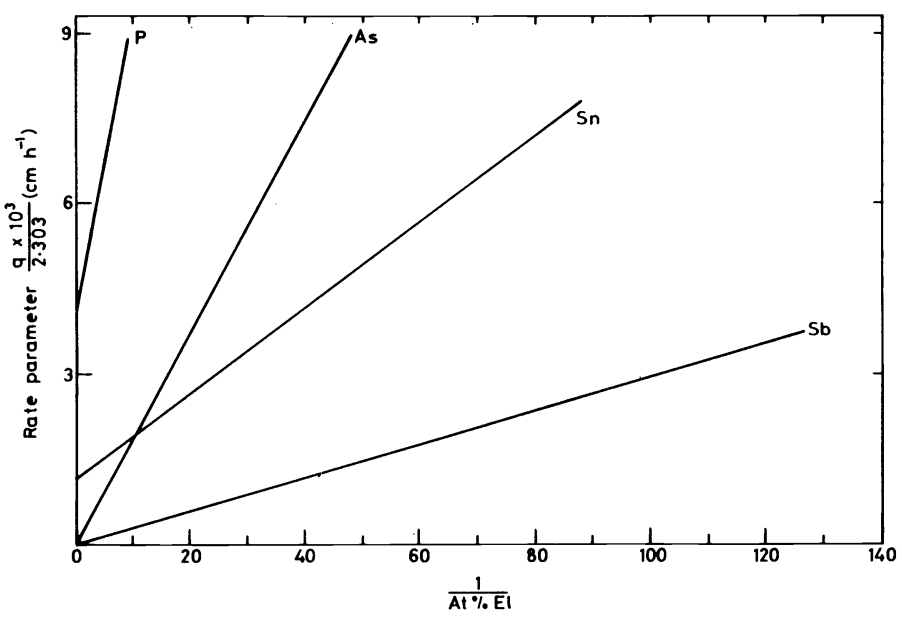

Fig. 9 Relative effects of several surfactant elements on the rates of surface nitridation of iron (from Ref 11)

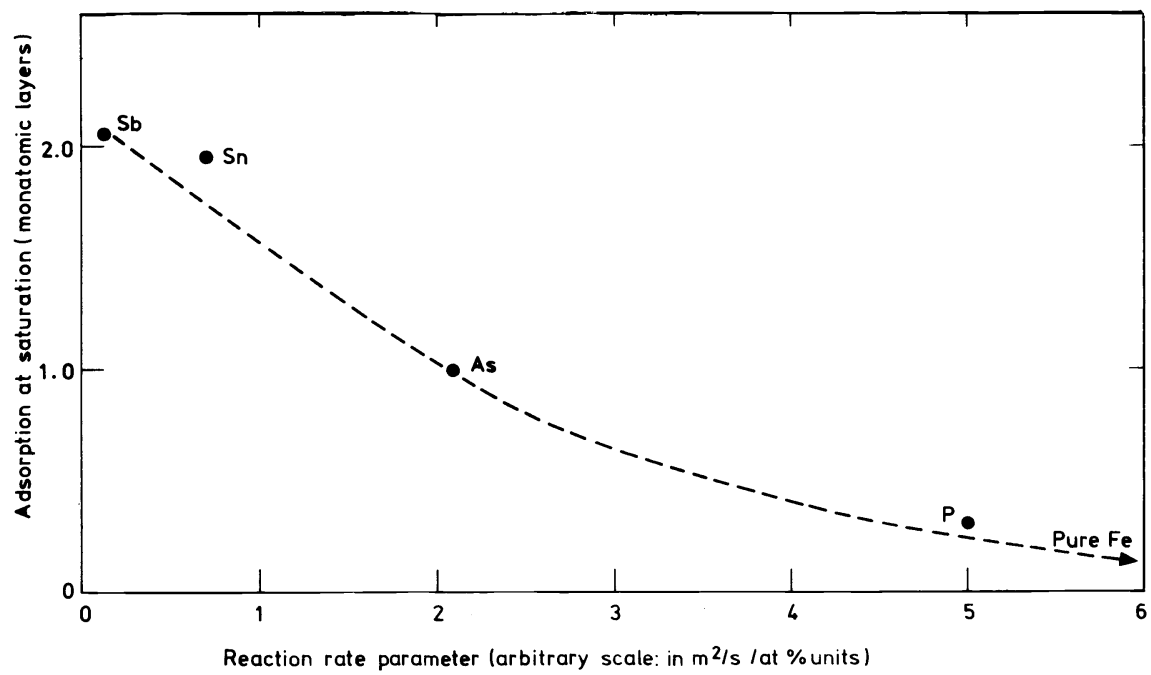

Fig. 10 Relation between the reaction rate parameters for surface nitridation of iron in the presence of various species and the estimated surface adsorption at saturation (monatomic layers) 
The data for the second category have been plotted in Fig. 10 where the predicted surface enrichment levels (based on experience and calculations in this Laboratory) are compared with the inhibitive values based on data in Fig. 9. The indications are that the species which procure a strong inhibitory effect during nitridation and carburisation also have high surface coverage. The converse, that is, any surface active species will procure an effect does not hold. It is interesting to note that compounds from this group have long been identified as potent catalytic poisons.

The relation of Fig. 10, albeit semi-quantitative, is relevant to the proposed mechanisms for the surface inhibition effect. The data in general support the 'bottle neck' theory, that is, with a high surface coverage by the poisoning species, only a small fraction of the free metal surface is available to provide the suitable sites for the catalytic dissociation step and the subsequent passage of the nitrogen atoms through the surface. That is, the paucity of sites available for the gas atoms to penetrate into the interior may create a sort of 'pile up' of nitrogen atoms waiting to cross into the metal substrate. In this mechanism, the bottle neck represents the rate determining step for the reaction. A detailed treatment of this notion supplies a specific (5ate equation which (fayy) analysed the absorption of nitrogen into liquid iron in the presence of oxygen or selenium and showed that the apparent rate constant for the reaction is directly proportional to $\left(1-X_{s}\right)$ where $X_{s}$ is the fractional coverage of the surface by the poisoning element.

In the category of Group VI poisons, however, such a mechanism cannot account for the fact that a saturation adsorption of oxygen at sub-monolayer levels is achieved and maintained over a very wide range of oxygen partial pressure, over which range the measurements on nitridation and carburisation rates indicate a continuing inhibition of the reaction with increasing oxygen or sulphur partial pressures. For this category, a different inhibition mechanism probably operates.

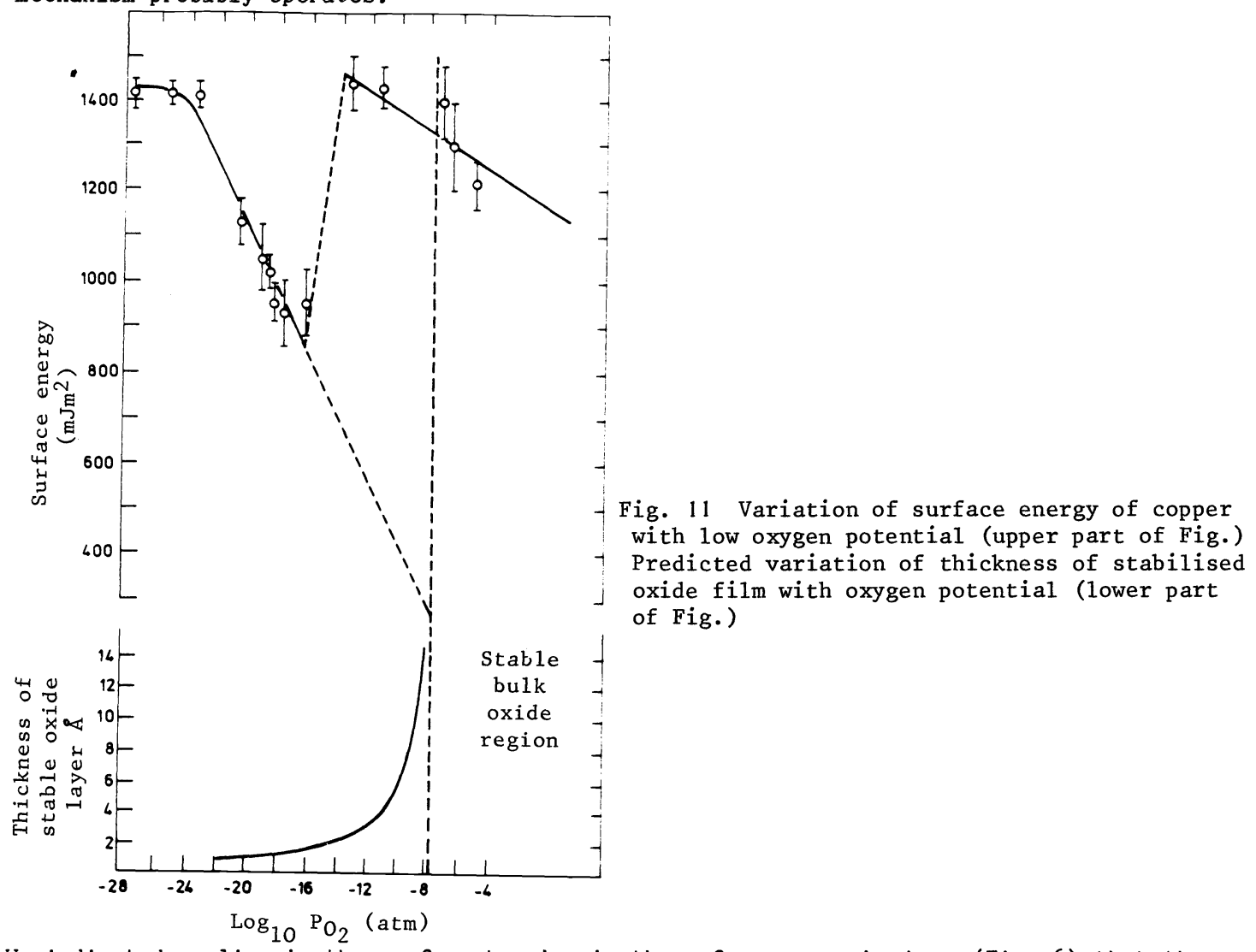

We indicated earlier in the surface tension isotherm for oxygen in iron (Fig. 6) that the isotherms at low partial pressures of oxygen follow the conventional Gibbsian form but anomalous behaviour was observed at higher oxygen potentials which are considerably lower than those predicted for bulk oxide formation. Such a discontinuity for the copper/oxygen isotherm at $927{ }^{\circ} \mathrm{C}$ is reproduced in the upper part of Fig. 11 (14); here at Log $\mathrm{pO}_{2}=-15$, there is a discontinuity in the isotherm although, according to bulk thermochemical data the oxidation should not start until Log $\mathrm{pO}_{2}=-7$. According to the Gibbs theorem, the surface energy should continue to fall linearly with Log po, until some other surface phenomenon occurs such as, for example, compound formation. We believe that in this instance and similarly for oxygen on iron, a surface compound, probably a protoxide, is formed. Calculations indicate that the optimum thickness of a quasi-oxide film increases at first slowly with increasing $\mathrm{pO}_{2}$ and then very rapidly as the $\mathrm{pO}_{2}$ 
approaches the value for bulk oxidation. This is computed on the assumption that the film possesses thermodynamic properties such as interfacial free energy and it is this free energy which allows the thin film to form in a stable manner at the low oxygen potentials. At a $\log \mathrm{pO}_{2}$ range between -20 and -10 , the thickness of this hypothetical film is so small that it can hardly be considered to be a separate oxide film. This leads to the view that in normal chemical reactions at high temperature, a precursor compound film exists at oxygen potentials well below those normally computed from bulk thermochemical data. This is because the existing metal surface is replaced by two interfaces possessing a lower total interfacial free energy. In these conditions adsorption takes the form of a surface two-dimensional phase or some ordered arrangement of adsorbed species and bulk species. We note that in recent studies using Low Energy Electron Diffraction the existence of surface two-dimensional adsorbed phases has now been demonstrated. A detailed description of the mechanism of nitridation and carburisation inhibition - at least for Group VI poisons should take into account the existence of such stable phases.

\section{GRAIN BOUNDARIES OR TYPE B INTERFACES}

Intercrystalline boundaries shown as B-type in Fig. 1 separate crystals of different crystallographic orientation. These extremely important interfaces are known to control many mechanical properties of engineering materials which depend on the cohesion at these interfaces. They are also important for metallurgical rate processes, because being the paths for the rapid transport of matter, they should be rate controlling in particular in those processes relating to regimes of temperature of about $0.5 \mathrm{~T}$ or below. This includes phenomena such as the growth or elimination of cavities or gas pockets situated on boundaries and also the transport of matter along the boundaries of metal oxides in the course of oxidation.

The adsorption processes at internal interfaces described earlier should affect the rate of diffusion of matter. Here we refer to recent developments in understanding the theory of segregation controlled mass transport at grain boundaries and consider a specific application, namely, the rate of sintering in the presence of surface active elements.

From the simplistic consideration that an internal interface is a structural zone with a low volume density of matter compared with the bulk, it should be expected that the activation energy barrier for self-diffusion should be less than for bulk crystalline matter. In a pure polycrystal, measurements indeed show that this is the case. Now extend this naive model by introducing adsorbing solute species to fill up the empty spaces in the structure of the boundary, in effect permitting the sites of high compression and tension to be relaxed, thus reducing the free energy and increasing the volume density. From the diffusional point of view, the material in the boundary zone approximates more closely the character of the material in the bulk. Hence from these general considerations, segregation should increase the activation energy for grain boundary diffusion, compared with that in a pristine boundary. Again, from available data, this is indeed the case.

Recently, we have developed analytically this notion and have derived a rigorous expression linking the change in diffusivity to the amount adsorbed at the poundary. The details of this have been published in the paper by Bernardini et al $(1982)(15)$ and a more general review can be found in the fpapter by Hondros and Seah (1984) . Starting from the theory of Borisov et al $(1964)(16)$ who derived a fundamental relation between grain boundary free energy and grain boundary diffusivity, the authors transformed the equation by introducing the amount of segregation $\Gamma$ in place of the grain boundary free energy $\gamma$. For a binary system in which grain boundary segregation of the second component takes place, the treatment yields the equation:

$$
\frac{D_{b}^{*}}{D_{v}^{*}}=\frac{D_{b}}{D_{v}}\left[1-\frac{\Gamma_{b}}{\Gamma_{b}^{\circ}}\right] \frac{2 a_{s v}^{2}}{m a_{s u}^{2}}
$$

where $D_{*}^{*}$ is cus oratil boundary self diffusion coefficient in the presence of segregation, $D^{*}$ is the coefficient due to the presence of the solute in the bulk, $D_{p}$ and $D_{y}$ are the corresponding values respectively in the pure solvent, $a$ is the atomic size of the solute in the particular solvent and $m$ is the quantity retained from the Borisov et al treatment and which relates to the number of atom layers in the boundary.

This equation has now been confirmed experimentally for tin additions in iron, by comparing the measurements of grain boundary diffusivity with those expected from the above equation, and knowing the amount of segregation by Auger Electron Spectroscopy on the exposed grain boundaries. An example from the data is shown in Fig. 12, which 
Indicates clearly how the grain boundary self diffusivity of iron decreases with increasing levels of tin segregated at the boundary from varying levels of tin in the bulk. On the same figure, the heavy line indicates the effect predicted by the above equation. This shows clearly good agreement for the dilute approximation in which the above derivation applies. This equation has been simplified further into the form:

$$
D_{b}^{*}=D_{b}\left[1+\left(b_{v}-\frac{2 a^{2}}{m a_{s u}^{2}}\right) \quad \beta_{b} \quad x_{c}\right]
$$

Here we have introduced the grain boundary enrichment factor $\beta$ which now expresses the effect of the segregation; and $b$ is a constant of the order of 10 to 100 for binary systems and expresses the dependence of volume diffusivity on the presence of a solute, $X$. The equation predicts that grain boundary diffusivity may be reduced or increased defending on the balance between the opposing trends implicit in $\gamma$ and $b$. In general, however, the effect of the high enrichment factor for strongly segregating species will dominate, that is, $\gamma$ is much greater than $b_{v}$, leading in most instances to a reduction in grain boundary diffusivity.

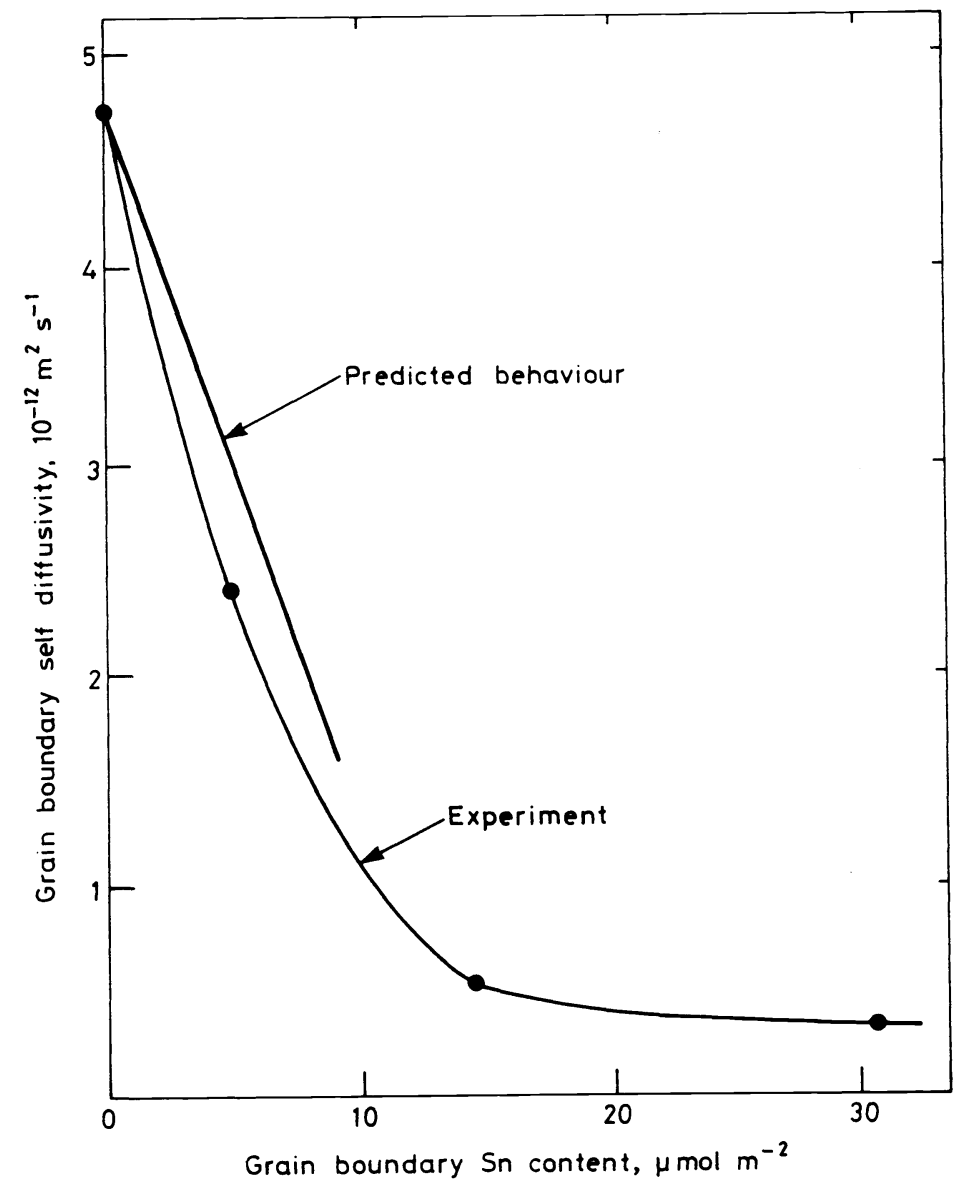

Fig. 12 Dependence of grain boundary self diffusivity of iron on bulk tin content: heavy line is the theoretically predicted relation (from Ref 15)

The above should have strong implications in metallurgical processes such as sintering, which requires essentially the elimination of a large volume of pore space from the mass of the material by diffusive processes which take place principally along grain boundaries. This has been confirffed in the system alumina in the presence of a dilute addition of yttria. Nanni et al $(17)$ measured the sintering rates at various temperatures for alumina in particulate form with and without the $\mathrm{Y}_{2} \mathrm{O}_{3}$ dopant which was predicted to be surface active. Fig. 13 shows a typical set of data from this work, for the rates of shrinkage of compacts of pure and doped alumina as a function of time at $1250{ }^{\circ} \mathrm{C}$. In the region to the right of the diagram where grain boundary transport operates, the yttria decreases the sintering rate compared with that of pure alumina. The activation energy for 
sinteriing was measurably increased by these yttria additions. Independent studies using Auger Electron Spectroscopy on fractured grain boundary surfaces of partially sintered material indicate that the yttria does segregate to the grain boundaries as predicted. Using an earlier form of Equation (6), it was proved that the levels of segregation measured here can indeed reduce the grain boundary diffusivity by the amounts required to sustain the decrease in sintering rates according to the 'Coble' model of sintering.

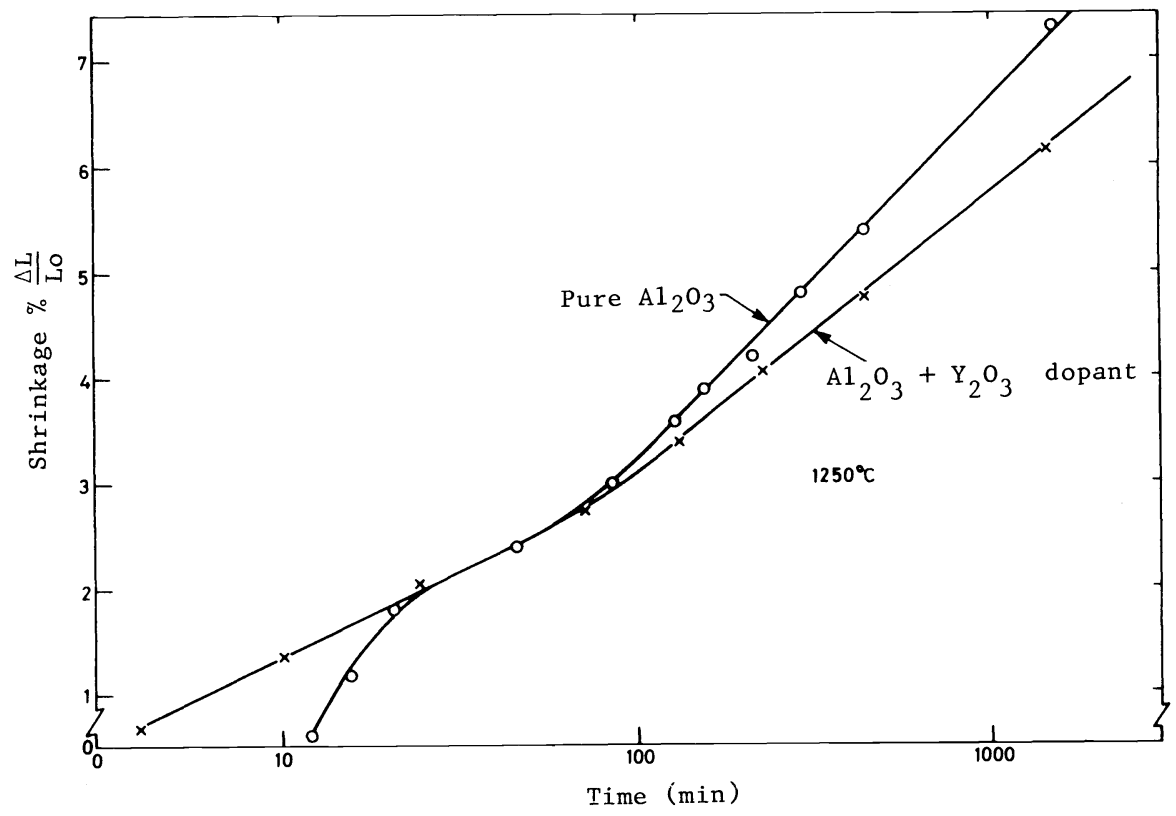

Fig. 13 Comparison of the rates of dilatation of sintering compacts of pure and yttrium-doped $\mathrm{Al}_{23}$ (from Ref 17)

\section{INTERFACE ADSORPTION AND OXIDATION INHIBITION}

Important benefits can be obtained by improving the corrosion resistance of components that must operate under stress at high temperatures. There are no simple criteria for improved oxidation resistance, particularly in complex service environments. Here mechanical damage of the oxide may occur through stresses arising from thermal expansion mismatch between oxide and substrate during temperature excursions, or again through erosion of the surfaces by high velocity gases. Although we consider this subject in terms of controlled chemical reactivity of the surface, in order to improve oxidation resistance it is not simply a matter of reducing the kinetics of oxide growth. It is equally necessary to ensure that the oxide has good adherence to the substrate so as to provide a diffusion barrier between the environment and the metal, as opposed to the periodical mechanical spalling of the oxide which exposes new metal surface for rapid oxidation. Although the precise atomistic mechanisms of improved oxidation resistance are the subject of debate, a range of additives have been identified by empirical procedures which improve the oxidation resistance of certain important engineering alloys. These dopanta generally segregate to interfaces of the type $C$ and $D$ in the schematic diagram of Fig. 1 and consequently influence the oxidation reaction.

Here we supply examples which relate both to metal/metal oxide interfaces and also to metal oxide surface/vapour interfaces. These cases are rare, but they provide strong evidence of adsorption-induced oxidation inhibition and suggest scope for improvements in understanding in this subject area.

Free surface oxidation inhibition

In the same way that reactions involving the gasification of the surface layers of metals can be retarded by the presence of surface active species, oxidation reactions may be impaired by adsorption at the free oxide surface.

In one example, we note the strong effect of certain elements at trace levels, in particular boron, in improving remarkably the oxidation resistance of certain alloy steels containing chromium, by increasing the time to 'breakaway' oxidation. Thus in an alloy $18 f$
$\mathrm{Fe}-10 \% \mathrm{Cr}$ heated for 28 days at $600{ }^{\circ} \mathrm{C}$ in an atmosphere containing traces of boron, Lea 
showed that the boron was concentrated at the outer surface of the oxide scale. An example of the concentration profiles produced by combining Auger Electron Spectroscopy with sputter etching in the oxide layer is shown in Fig. 14. Here, the origin at the absissa scale corresponds to the plane of the free oxide surface. The levels of boron are high at the outer surface and fall rapidly at depths of only a few atom distances. Furthermore, the high boron content of the outer surface is accompanied by chromium enrichment.

It is remarkable how small traces of boron at the parts per million level in the vapour phase can produce dramatic effects on the oxidation resistance of this alloy. The inhibition mechanism appears to be related to the presence of boron in close association with chromium at the outer oxide surface. As in the previous considerations of two-dimensional phase formation, the speculation here is that $0-B-0$ covalent bonds are formed in the outer oxide surface structure and this skin of stable compound should act as a barrier to the transfer of oxygen ions into the substrate. In turn, this protective film should allow the slowly diffusing chromium from the alloy to reach the free oxide surface to act as an oxidising inhibitor. These notions relating to reaction inhibition may be applicable to other oxidising systems; here in particular, the use of X-ray Photo-electron Spectroscopy should provide the additional bonding information required for a more thorough description of the microchemistry of the surface which leads to the inhibitory effect.

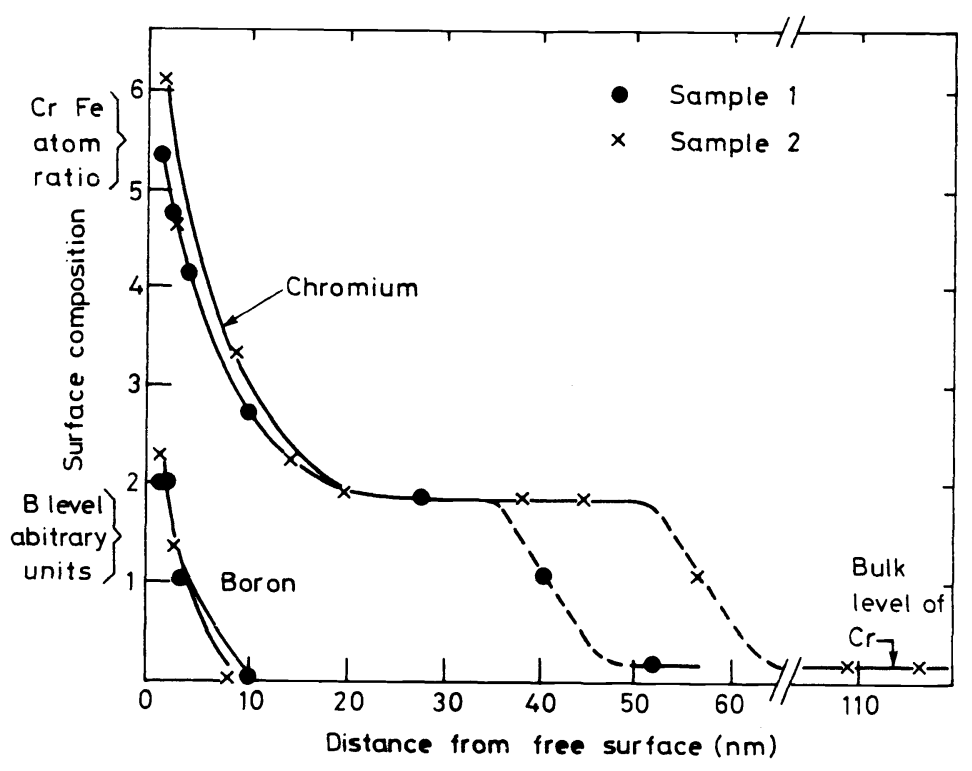

Fig. 14 Depth composition profiles on oxidised iron-chromium alloy showing the concentration of boron trace impurity at the surface (from Ref 18)

Oxidation inhibition by adsorption at the metal/metal oxide interface

There are cases of oxidation inhibition resulting from adsorbed dopant films at the alloy/scale interface, which act as a barrier to the transfer of species required for the reaction to proceed. Such barrier films may form by kinetic processes or they may occur as an equilibrium adsorptign process. Thus, in the case of iron/silicon alloys, it was predicted by Atkinson that the kinetics of oxidation would lead to a discontinuous complex film (at) the alloy/scale interface. This was later confirmed experimentally by Moseley et al $(20)$ using X-ray Photo-electron Spectroscopy on the oxide scale stripped from the alloy by a chemical process. From peak energy separation data, it was suggested that the silicon rich layer at the interface comprised oxygen deficient $\mathrm{SiO}_{2}$.

In another class of alloy, the nickel/chromium based superalloys which are important as turbine components, a large experimental effort has gone into the study of the effects of trace additions of elements such as yttrium, cerium and other rare earth metals on the oxidation resistance. Different alloys have different responses to these additives. This complex subject matter has been reviewed by Whittle and Stringer (2T) who discuss the various possible mechanisms for the reaction inhibition. 


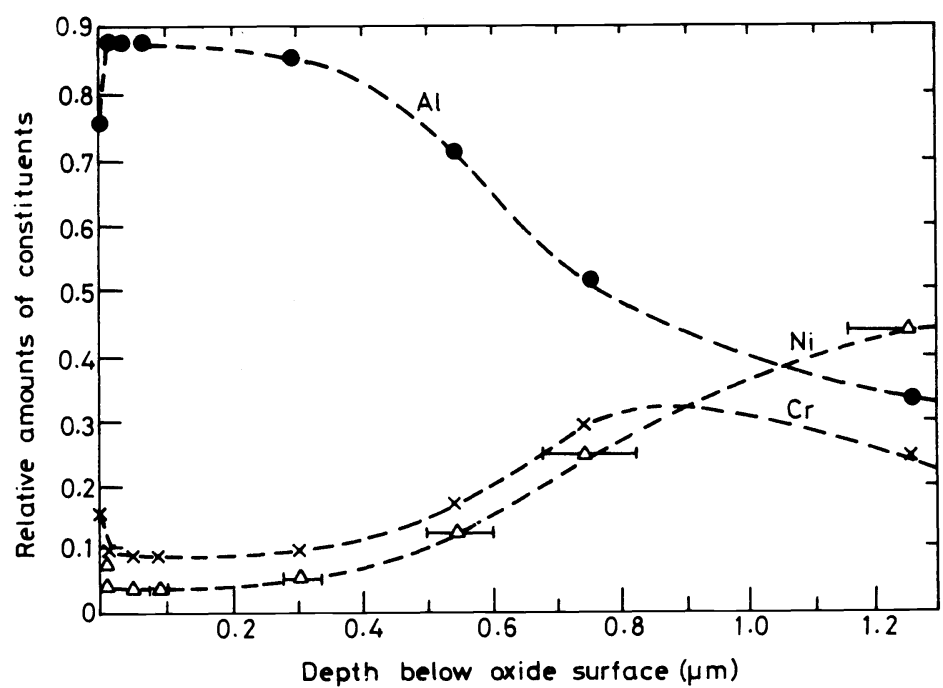

(a)

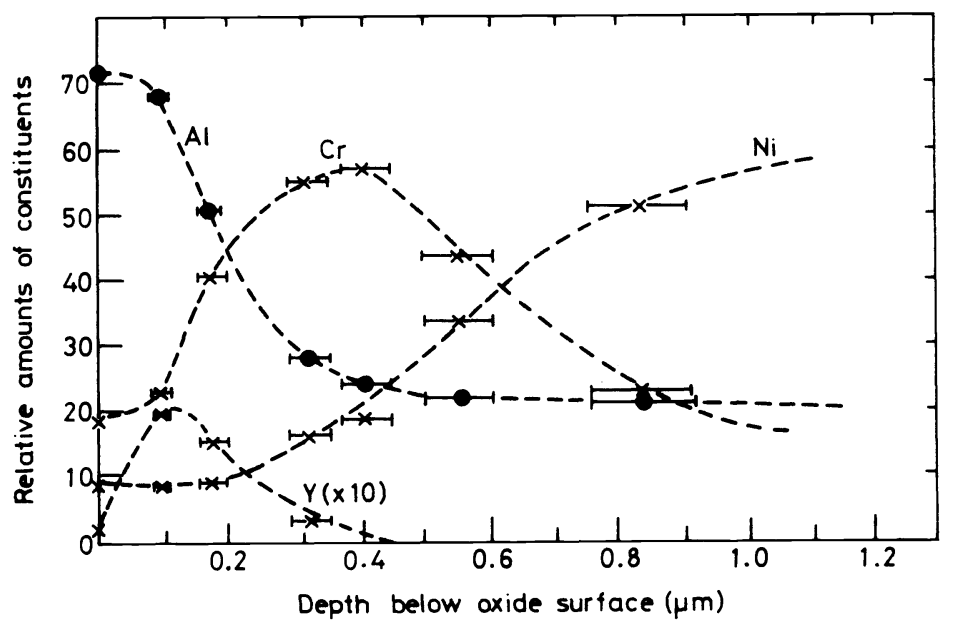

(b)

Fig. 15 Depth composition for the initial oxide formed on $\gamma-\gamma^{\prime}-\mathrm{Cr}_{2} \mathrm{C}_{2}$ alloy composite

(a) basic aIloy

(b) yttrium doped alloy

In the case of the in situ composite superalloy $\gamma-\gamma^{\prime}-\mathrm{Cr}_{3} \mathrm{C}_{2}$, the effect of doping the alloy with yttrium additions up to $0.5 \%$ was a remarkable $\frac{3}{2}$ eduction in the weight change during thermal cycling to temperature maxima of $1200{ }^{\circ} \mathrm{C}(2)$. Microstructural examination showed that the pure alloy has a thick, complex oxide layer which may spall periodically, whereas the yttrium-doped alloy has a very thin tenacious oxide with no evidence of spalling. In both samples, the initial stable oxide formed on this alloy is $\mathrm{Al}_{2} \mathrm{O}_{3}$. Using Auger Electron Spectroscopy in combination with sputter profiling, differences $3^{\circ}$ in the compositional distribution of the various elements are indicated in Figs 15a and 15b. In the undoped sample, the outer oxide scale is rich in aluminium, consistent with the presence of $\mathrm{Al}_{2} \mathrm{O}_{3}$ and there are smaller amounts of nickel and chromium. With increasing depth, the varlous elemental constituents approach the levels of the bulk. On the other hand, in the doped sample, there are clear differences in the distribution of aluminium and chromium, and the yttrium rises rapidly from zero at the oxide surface, reaching a peak level in the oxide bulk at a depth corresponding to the position of the oxide/metal interface. This is evidence that the yttrium segregates to the oxide/metal interface in addition to the $\mathrm{Al}_{2} \mathrm{O}_{3}$ grain boundaries discussed earlier.

The mechanism for the strong inhibitive effect of yttrium is attributed to both the reduction in grain boundary mass transport due to the segregation of the yttrium to the 
grain boundaries (see previous section) and also to an indirect effect of the yttrium present at the metal/metal oxide interface. The fact that there is less spalling in the doped alloy suggests that the oxide/metal adhesion has been improved and the evidence of inhibited grain growth in the oxide scale suggests that any expansion mismatch can be accomodated by plastic flow of the oxide by grain boundary sliding rather than by de-cohesion at the metal oxide/metal interface.

\section{REACTION INHIBITION AT PHASE INTERFACES}

Above we considered some effects on oxidation due to adsorption at a two-phase interface. A similar sort of interface is presented where a second-phase particle, such as a precipitate, is embedded in a metallic matrix, indicated as type $C$ in Fig. 1. In metallurgical practice, the maintenance of high temperature mechanical properties in certain alloys depends on the size stability of a fine dispersion of second-phase particles. Such properties could be impaired if, during the service temperature or during previous thermal treatments, the fine particles coarsen into a less dense distribution of larger particles. This coarsening or ripening process is driven by the thermodynamics of interfaces, in effect the tendency to reduce the total interfacial area and therefore the total free energy associated with these particles. The mechanism of this ripening process has been studied over many years and the rate determining step is normally the diffusivity of the species through the bulk metal. However, in the presence of a strongly bound interfacial adsorbate at the particle/metal interface, the atom transfer step may become rate determining, leading to a significant retardation in the ripening kinetics.

The starting point for considerations of ripening reactions is the Lifshitz/Wagner (23) equation for the volume diffusion control growth of a dispersion of particles, in the form:

$$
r^{3}-r_{0}^{3}=\frac{B_{1} \gamma V^{2} C_{0} D_{V}}{R T}\left(t-t_{0}\right)
$$

where $r$ is the mean particle radius, $V$ the molar volume of precipitates, $C_{\circ}$ the molar concentration of solute in equilibrium with a particle of infinite size, ${ }^{\circ}{ }_{1} a$ numerical parameter which can be varied to include a term for the shape of the precipitates. Adsorption to such an interface should reduce the interfacial energy $\gamma$ typically by about $50 \%$. However, much larger inhibitions in coarsening behaviour have been observed and since $\gamma$ enters Equation (7) in a monotonic way, a reduction in interfacial energy cannot itself account for the observations.

There is now much indirect information for the adsorption of alloying elements to such interfaces, which leads to changes in the ripening reaction. Thus, Boyd and Nicholson showed that $0.1 \%$ cadmium added to an aluminium $/ 4 \%$ copper alloy produced a large reduction in the ageing kinetics of $\gamma$, precipitates at $200{ }^{\circ} \mathrm{C}$. A measured reduction in the interfacial energy between the particle and the metal implied that cadmium adsorbed at this interface. In the meantime, the subject matter has advanced considerably in the study of the ripening characteristics of $\gamma^{\prime} / \mathrm{Fe}_{4} \mathrm{~N}$ precipitates in an iron/nitrogen alloy, in the many contributions to this subject by Grieveson and his collaborators. Earlier they indicated that low levels of oxygen present in the bulk inhibits the rate of coarsening of the nitrides. This work has now been extended and there is firm evidence that species such as antimony, phosphorus and tin also inhibit coarsening. That this effect is quite significant is illustrated in the recent data for the coarsening of nitride precipitates in an iron $/ 0.06 \% \mathrm{~N}$ alloy obtained by Miller et al . Here, increasing the bulk tin content up to $0.19 \%$ reduces the mean nitride particle coarsening rate by an order of magnitude compared with the behaviour in pure iron. This data is illustrated in Fig. 16. 


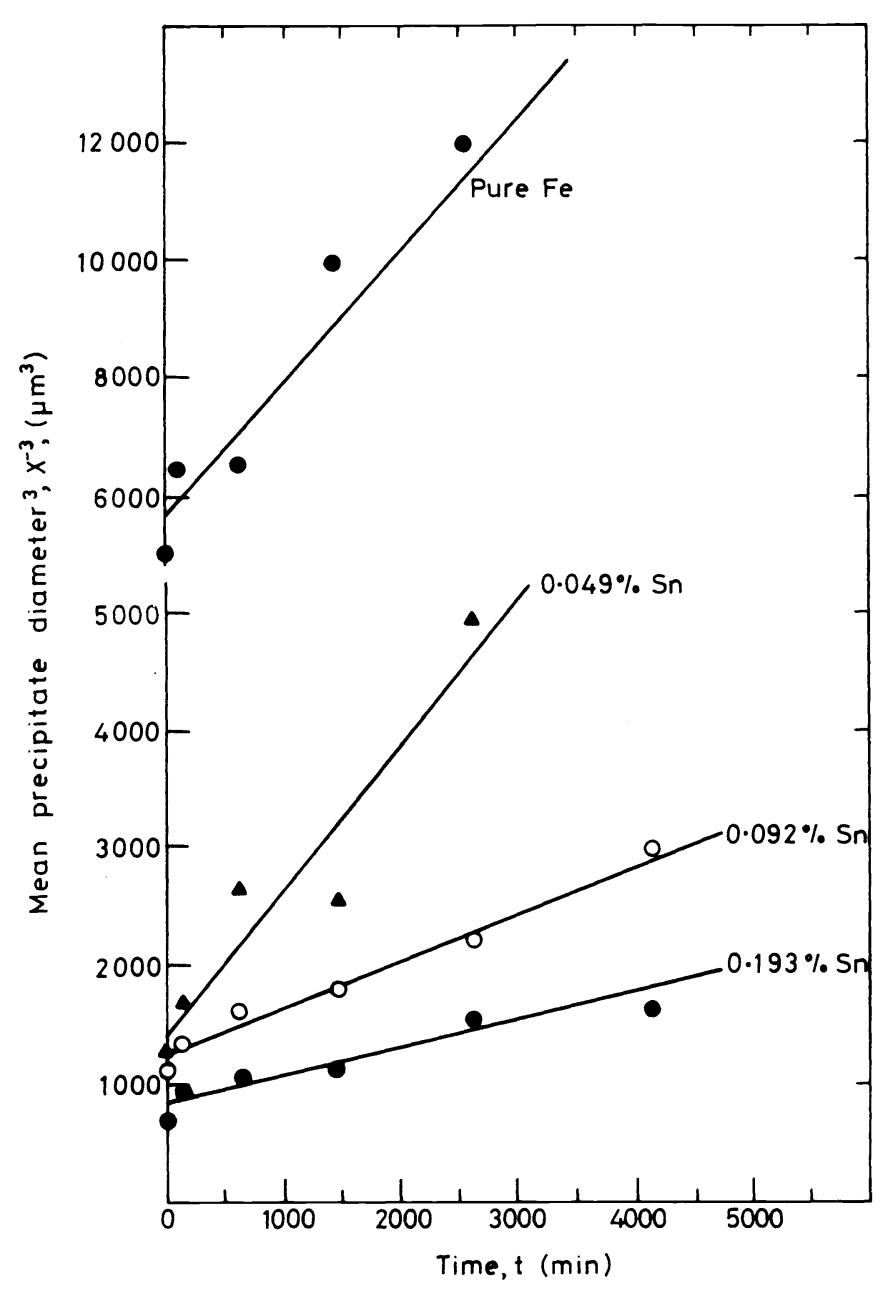

Fig. 16 Rate of coarsening of nitride precipitates in iron in the presence of tin additive

Where the reaction is controlled at the interface, Equation 7 takes the form,

$$
r^{2}-r_{0}^{2}=\frac{\mathrm{B}_{2} \gamma \mathrm{V}^{2} \mathrm{C}_{0} \mathrm{Q}}{\mathrm{RT}}\left(t-t_{0}\right)
$$

where $Q$ is a reaction rate parameter associated with the ease of transferring species across the interfaci. Thus, the particle coarsening theory predicts that the mean particle size varies with $t^{\frac{1}{3}}$ for volume diffusion control and with $t^{\frac{1}{2}}$ for interface control. In most studies of this nature, it has not been possible to make a clear distinction in mechanisms on the basis of time-dependent growth behaviour, because these functions are relatively insensitive and the data could be fitted to either function within experimental error. The theory also predicts a time invariant size distribution profile, depending on the growth mechanism. The theoretical distribution function $\rho$ h is shown in Fig. 17, where $\rho^{2} h=\frac{f(X, t) X}{Z}$ and $X$ is the mean particle diameter; $f(X, T)$ is the number of particles in each size category and $z$ the number of particles per unit volume. This distribution function predicts a break at $\frac{X}{X^{*}}=1.5$ for diffusion growth and $\frac{X^{*}}{X^{*}}=2.0$ for interface control. The data of Miller are also shown in Fig. 17 for the growth of $\mathrm{Fe}_{4} \mathrm{~N}$ particles in iron containing tin at the levels indicated. The results can be more closely attributed to the interface control model. 

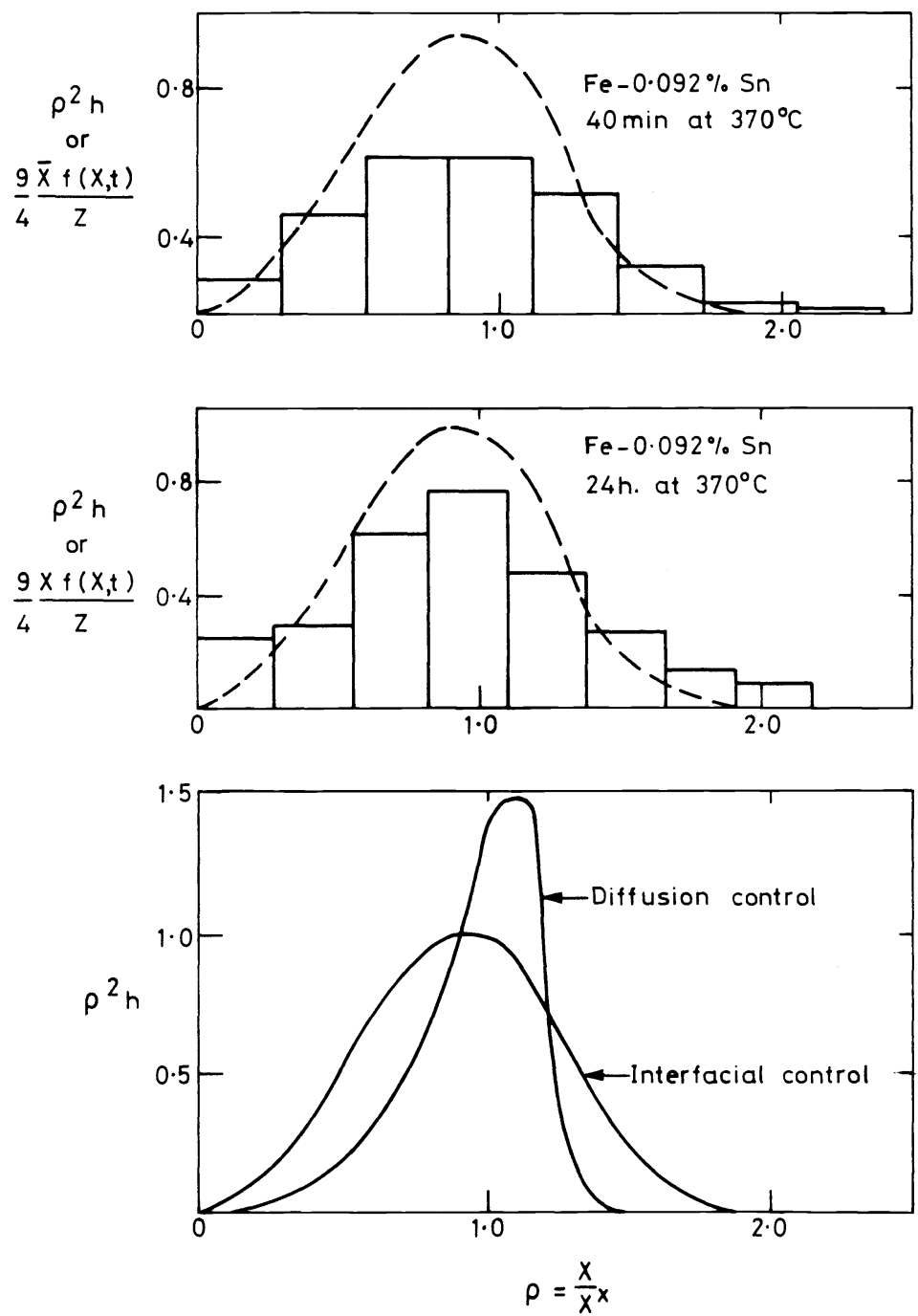

Fig. 17 Size distribution function for nitride precipitates in iron in the presence of tin additive: comparison with the theoretical functions for diffusion and interface controlled ripening (lower part of Fig.) (from Ref 11)

For the ripening characteristics in actual commercial nickel based alloys which (25) rate between $500-1000{ }^{\circ} \mathrm{C}$, in a recent analysis of data on a number of systems, McLean ${ }^{25}$ has concluded that volume diffusion control operates over a considerable time span and that the Liftshitz/Wagner equation correctly describes the ripening behaviour. He foresees, however, that whereas such behaviour is expected to proceed over a much longer time range, there is a possibility of the gradual poisoning of the particle interfaces by impurities, allowing interface reaction control to ensue. Whereas certain impurities such as sulphur, selenium and oxygen have very strong binding energies at interfaces, these are uniikely to operate as poisoning elements for the volume diffusion control reaction because of the large concentration that is required. This does not conflict with the observations made above that additives which are known to be interfacially active and in reasonable concentrations may effect changes to the ripening behaviour, converting a volume diffusion control process to one of interface reaction control.

Acknowledgements - The author thanks Professor P Grieveson of Imperial College for access to unpublished material and to $\mathrm{Dr} C$ Lea and $\mathrm{Dr} M \mathrm{P}$ Seah for fruitful discussions. 


\section{REFERENCES}

(1) E D Hondros and M P Seah, Chapter 13 in Physical Metallurgy, 3rd Edition, $R$ W Cahn and $P$ Haasen, eds., North-Holland Publishing Company, (1984).

(2) E D Hondros, Proc. Roy. Soc. A, 286, 479 (1965).

(3) D McLean, Grain Boundaries in Metals, Clarendon Press, Oxford (1957).

(4) E D Hondros and M P Seah, Metallurg. Trans. A8, 1363.

(5) E T Turkdogan and P Grieveson, J. Electrochem. Soc., 114, 59 (1967).

(6) L S Darken and E T Turkdogan, Heterogeneous Kinetics at Elevated Temperatures, Plenum Press, NY, 25-101 (1970).

(7) R J Fruehan, Metallurg. Trans., $\underline{3}, 1447$ (1972).

(8) E D Hondros, Acta Metallurg., 16, 1377 (1968).

(9) H J Grabke, W Paulitschke, G Tauber and H Viefhaus, Surface Sci., 63, 377 (1977).

(10) $P$ Hayes and P Grieveson, Acta Metall., 23, 937 (1975).

(11) D Miller, R Pomfret and P Grieveson (unpublished work), University of Strathclyde, Dpt of Metallurgy, (1979).

(12) F Togashi and T Nishizawa, "Effect of Tin and Antimony on the Rate of Decarburisation of Austenite in wet Hydrogen", personal communication.

(13) G R Belton, Metallurg. Trans., 7B, 35 (1976).

(14) M McLean and E D Hondros, J. Mater. Sci., 8, 349 (1973).

(15) J Bernardini, P Gas, E D Hondros and M P Seah, Proc Roy Soc A, 379, 159 (1982).

(16) V I Borisov, V M Golikov and G V Scherbedinkskiy, Phys Met and Metallog, 17, 80 (1964).

(17) P Nanni, C T H Stoddart and E D Hondros, Mater Chem, 1, 297 (1976).

(18) C Lea, Metals Sci, 13, 301 (1979).

(19) A Atkinson, AERE Report R9960, UKAEA Harwell, Feb 1981.

(20) P T Moseley, G Tappin and J C Riviere, AERE Report R10042, UKAEA Harwell, Feb 1981.

(21) D P Whittle and J Stringer, Phil Trans R Soc Lond, A295, 309.

(22) E Bullock, C Lea and M McLean, Metal Sci, 373 June (1979).

(23) I M Lifshitz and V V Sloyozov, Phys Chem Solids, 19, 35 (1961).

(24) J D Boyd and R B Nicholson, Acta Metall, 19, 1379 (1971).

(25) D McLean, Metall Sci., 18, 249(1984). 